\title{
Commuting maps on alternative rings 1
}

\author{
Bruno Leonardo Macedo Ferreira \\ Federal University of Technology, \\ Professora Laura Pacheco Bastos Avenue, 800, \\ 85053-510, Guarapuava, Brazil. \\ brunoferreira@utfpr.edu.br \\ Ivan Kaygorodov \\ Federal University of ABC, \\ dos Estados Avenue, 5001, \\ 09210-580, Santo André, Brazil. \\ kaygorodov.ivan@gmail.com
}

Keywords: Commuting maps; alternative rings AMS: 17D05, 47B47.

\begin{abstract}
Suppose $\mathfrak{R}$ is a 2,3-torsion free unital alternative ring having an idempotent element $e_{1}\left(e_{2}=1-e_{1}\right)$ which satisfies $x \Re \cdot e_{i}=\{0\} \Rightarrow$ $x=0(i=1,2)$. In this paper, we aim to characterize the commuting maps. Let $\varphi$ be a commuting map of $\mathfrak{R}$ so it is shown that $\varphi(x)=$ $z x+\Xi(x)$ for all $x \in \mathfrak{R}$, where $z \in \mathcal{Z}(\Re)$ and $\Xi$ is an additive map from $\mathfrak{R}$ into $\mathcal{Z}(\mathfrak{R})$. As a consequence a characterization of anti-commuting maps is obtained and we provide as an application, a characterization of commuting maps on von Neumann algebras relative alternative $C^{*}$ algebra with no central summands of type $I_{1}$.
\end{abstract}

\section{Introduction}

Let $\mathfrak{R}$ be a unital ring not necessarily associative or commutative and consider the following convention for its multiplication operation: $x y \cdot z=(x y) z$ and $x \cdot y z=x(y z)$ for $x, y, z \in \mathfrak{R}$, to reduce the number of parentheses. We denote the associator of $\mathfrak{R}$ by $(x, y, z)=x y \cdot z-x \cdot y z$ for $x, y, z \in \mathfrak{R}$. And $[x, y]=x y-y x$ is the usual Lie product of $x$ and $y$, with $x, y \in \mathfrak{R}$.

\footnotetext{
1 This work was supported by FAPESP 19/03655-4; CNPq 302980/2019-9; RFBR 20-01-00030.
} 
Let be $\varphi: \mathfrak{R} \rightarrow \mathfrak{R}$ a additive map of $\mathfrak{R}$ into $\mathfrak{R}$. We call $\varphi$ a commuting map of $\mathfrak{R}$ into $\mathfrak{R}$ if for all $x \in \mathfrak{R}$ :

$$
[\varphi(x), x]=0 .
$$

Divinsky at [8] started a study on commuting maps, he proved that a simple Artinian ring is commutative if it has a commuting automorphism different from the identity map. In the face of this study, some mathematicians began to investigate the problem of characterizing some maps on associative rings. Bresar in 3] proved that, if $F$ is a commuting additive map from a von Neumann algebra $M$ into itself, then there exist $Z \in \mathcal{Z}(M)$ and an additive map $h: M \rightarrow \mathcal{Z}(M)$ such that $F(A)=Z A+h(A)$ for all $A \in M$. Later, Bresar [5] gave the same characterization of commuting additive maps on prime rings. For the reader interested in other results about characterization of maps on associative rings and algebras, see refs. [4, 6, 7, 19, 20] and the references therein. For the class of alternative rings and algebras studies about many linear and non-linear maps have been an interesting and active research topic recently, we can quote [9, 10, 12, 11, 13, 18, 14. An important class of 8-dimensional Cayley algebras (or Cayley-Dickson algebras, the prototype having been discovered in 1845 by Cayley and later generalized by Dickson) is so called octonions algebra a class of alternative algebras which are not associative. Given any algebra $\mathfrak{A}$ of dimension $n$ with an $*$-involution an algebra $\mathfrak{B}$ of dimension $2 n$ was constructed by Albert $[1$ as $\mathfrak{B}=\{x+y i \mid x, y \in \mathfrak{A}\}$. Albert proved that if $\mathfrak{B}$ is associative, then $\mathfrak{A}$ is commutative and that $\mathfrak{A}$ is associative if and only if the elements of $\mathfrak{B}$ satisfy $(u, u, v)=0=(v, u, u)$. Given this we have the following.

A ring $\mathfrak{R}$ is said to be alternative if $(x, x, y)=0=(y, x, x)$ for all $x, y \in \mathfrak{R}$. One easily sees that any associative ring is an alternative ring. A ring $\mathfrak{R}$ is called $k$-torsion free if $k x=0$ implies $x=0$, for any $x \in \mathfrak{R}$, where $k \in \mathbb{Z}, k>0$, and prime if $\mathfrak{A} \mathfrak{B} \neq 0$ for any two nonzero ideals $\mathfrak{A}, \mathfrak{B} \subseteq \mathfrak{R}$. The nucleus of a ring $\mathfrak{R}$ is defined by

$$
\mathcal{N}(\mathfrak{R})=\{r \in \mathfrak{R} \mid(x, y, r)=(x, r, y)=(r, x, y)=0 \text { for all } x, y \in \mathfrak{R}\} .
$$

The commutative center of an ring $\mathfrak{R}$ is defined by

$$
\mathcal{Z}(\mathfrak{R})=\{r \in \mathfrak{R} \mid[r, x]=0 \text { for all } x \in \mathfrak{R}\} .
$$

The next result can be found in [10] 
Theorem 1. Let $\mathfrak{R}$ be a 3 -torsion free alternative ring. So $\mathfrak{R}$ is a prime ring if and only if $a \mathfrak{R} \cdot b=0$ (or $a \cdot \mathfrak{R} b=0$ ) implies $a=0$ or $b=0$ for $a, b \in \mathfrak{R}$.

A nonzero element $e_{1} \in \mathfrak{R}$ is called an idempotent if $e_{1} e_{1}=e_{1}$ and a nontrivial idempotent if it is an idempotent different from the multiplicative identity element of $\mathfrak{R}$. Let us consider $\mathfrak{R}$ an alternative ring and fix a nontrivial idempotent $e_{1} \in \mathfrak{R}$. Let $e_{2}: \mathfrak{R} \rightarrow \mathfrak{R}$ and $e_{2}^{\prime}: \mathfrak{R} \rightarrow \mathfrak{R}$ be linear operators given by $e_{2}(a)=a-e_{1} a$ and $e_{2}^{\prime}(a)=a-a e_{1}$. Clearly $e_{2}^{2}=e_{2}$, $\left(e_{2}^{\prime}\right)^{2}=e_{2}^{\prime}$ and we note that if $\mathfrak{R}$ has a unity, then we can consider $e_{2}=$ $1-e_{1} \in \mathfrak{R}$. Let us denote $e_{2}(a)$ by $e_{2} a$ and $e_{2}^{\prime}(a)$ by $a e_{2}$. It is easy to see that $e_{i} a \cdot e_{j}=e_{i} \cdot a e_{j}(i, j=1,2)$ for all $a \in \mathfrak{R}$. Then $\mathfrak{R}$ has a Peirce decomposition $\mathfrak{R}=\mathfrak{R}_{11} \oplus \mathfrak{R}_{12} \oplus \mathfrak{R}_{21} \oplus \mathfrak{R}_{22}$, where $\mathfrak{R}_{i j}=e_{i} \mathfrak{R} e_{j}(i, j=1,2)$ [17, satisfying the following multiplicative relations:

(i) $\mathfrak{R}_{i j} \mathfrak{R}_{j l} \subseteq \mathfrak{R}_{i l}(i, j, l=1,2)$;

(ii) $\mathfrak{R}_{i j} \mathfrak{R}_{i j} \subseteq \mathfrak{R}_{j i}(i, j=1,2)$;

(iii) $\mathfrak{R}_{i j} \mathfrak{R}_{k l}=0$, if $j \neq k$ and $(i, j) \neq(k, l),(i, j, k, l=1,2)$;

(iv) $x_{i j}^{2}=0$, for all $x_{i j} \in \mathfrak{R}_{i j}(i, j=1,2 ; i \neq j)$.

The notion of Peirce decomposition for alternative rings is similar to that one for associative rings. However, this similarity is restricted to its written form, not including its theoretical structure since Peirce decomposition for alternative rings is a generalization of that classical one for associative rings. Taking this fact into account, in the present paper in a certain way we generalize the main Bresar's Theorem [5] to the class of alternative rings.

As mentioned before, recently the problem of characterizing maps on nonassociative rings has been studied. Ferreira and Guzzo worked the characterization of multiplicative Lie derivation on alternative rings.

In [12], they investigated the additivity of Lie multiplicative map where did they get the following result.

Theorem 2. Let $\mathfrak{R}$ and $\mathfrak{R}^{\prime}$ be alternative rings. Suppose that $\mathfrak{R}$ is a ring containing a nontrivial idempotent $e_{1}$ which satisfies:

(i) If $\left[a_{11}+a_{22}, \mathfrak{R}_{12}\right]=0$, then $a_{11}+a_{22} \in \mathcal{Z}(\mathfrak{R})$,

(ii) If $\left[a_{11}+a_{22}, \mathfrak{R}_{21}\right]=0$, then $a_{11}+a_{22} \in \mathcal{Z}(\mathfrak{R})$. 
Then every Lie multiplicative bijection $\varphi$ of $\mathfrak{R}$ onto an arbitrary alternative ring $\mathfrak{R}^{\prime}$ is almost additive.

In another recent paper [11] they proved

Theorem 3. Let $\mathfrak{R}$ be a unital 2,3-torsion free alternative ring with nontrivial idempotents $e_{1}, e_{2}$ and with associated Peirce decomposition $\mathfrak{R}=$ $\mathfrak{R}_{11} \oplus \mathfrak{R}_{12} \oplus \mathfrak{R}_{21} \oplus \mathfrak{R}_{22}$. Suppose that $\mathfrak{R}$ satisfies the following conditions:

(1) If $x_{i j} \Re_{j i}=0$, then $x_{i j}=0(i \neq j)$;

(2) If $x_{11} \mathfrak{R}_{12}=0$ or $\mathfrak{R}_{21} x_{11}=0$, then $x_{11}=0$;

(3) If $\mathfrak{R}_{12} x_{22}=0$ or $x_{22} \Re_{21}=0$, then $x_{22}=0$;

(4) If $z \in \mathcal{Z}(\mathfrak{R})$ with $z \neq 0$, then $z \mathfrak{R}=\mathfrak{R}$.

Let $\mathfrak{D}: \mathfrak{R} \longrightarrow \mathfrak{R}$ be a multiplicative Lie derivation of $\mathfrak{R}$. Then $\mathfrak{D}$ is the form $\delta+\tau$, where $\delta$ is an additive derivation of $\mathfrak{R}$ and $\tau$ is a mapping from $\mathfrak{R}$ into the commutative centre $\mathcal{Z}(\mathfrak{R})$, which maps commutators into the zero if and only if

(a) $e_{2} \mathfrak{D}\left(\Re_{11}\right) e_{2} \subseteq \mathcal{Z}(\Re) e_{2}$,

(b) $e_{1} \mathfrak{D}\left(\mathfrak{R}_{22}\right) e_{1} \subseteq \mathcal{Z}(\mathfrak{R}) e_{1}$.

Inspired by the above mentioned results the purpose of the present paper is to consider the problem of characterizing commuting additive maps on alternative rings.

Let $\mathfrak{R}$ be a unity alternative ring and an idempotent element $e_{1}$, and let $\mathcal{Z}(\mathfrak{R})$ denote the commutative center of $\mathfrak{R}$. Assume that the characteristic of $\mathfrak{R}$ is not 2,3 and satisfies $x \Re \cdot e_{i}=0 \Rightarrow x=0(i=1,2)$. Let $\varphi$ : $\mathfrak{R} \rightarrow \mathfrak{R}$ be an additive map. We show that the following two statements are equivalent:

1. $\varphi$ is commuting;

2. there exist $z \in \mathcal{Z}(\mathfrak{R})$ and an additive map $\Xi: \mathfrak{R} \rightarrow \mathcal{Z}(\mathfrak{R})$ such that $\varphi(x)=z x+\Xi(x)$ for all $x \in \mathfrak{R}$.

As applications, a characterization of commuting additive maps on prime rings and von Neumann algebras relative alternative $C^{*}$-algebra with no central summands of type $I_{1}$ is obtained. 


\section{Main theorem}

We shall prove as follows the main result of this paper.

Theorem 4. Let $\mathfrak{R}$ be a unital 2,3-torsion free alternative ring. Assume that $\mathfrak{R}$ has a nontrivial idempotent $e_{1}$ with associated Peirce decomposition $\mathfrak{R}=\mathfrak{R}_{11} \oplus \mathfrak{R}_{12} \oplus \mathfrak{R}_{21} \oplus \mathfrak{R}_{22}$, such that $x \mathfrak{R} \cdot e_{i}=0 \Rightarrow x=0(i=1,2)$. Let $\varphi: \mathfrak{R} \rightarrow \mathfrak{R}$ be an additive map. Then the following statements are equivalent:

(\$) $\varphi$ is commuting;

(\$) There exist $z \in \mathcal{Z}(\mathfrak{R})$ and an additive map $\Xi: \mathfrak{R} \rightarrow \mathcal{Z}(\mathfrak{R})$ such that $\varphi(x)=z x+\Xi(x)$ for all $x \in \mathfrak{R}$.

It is obvious that prime alternative rings satisfy the assumptions " $x \Re$. $e_{1}=0 \Rightarrow x=0$ and $x \Re \cdot e_{2}=0 \Rightarrow x=0$ " by Theorem 1. So, we have the following result

Corollary 5. Let $\mathfrak{R}$ be a unital 2,3-torsion free prime alternative ring. Assume that $\mathfrak{R}$ has a nontrivial idempotent $e_{1}$ with associated Peirce decomposition $\mathfrak{R}=\mathfrak{R}_{11} \oplus \mathfrak{R}_{12} \oplus \mathfrak{R}_{21} \oplus \mathfrak{R}_{22}$. Let $\varphi: \mathfrak{R} \rightarrow \mathfrak{R}$ be an additive map. Then the following statements are equivalen:

(\$) $\varphi$ is commuting;

(\$) There exist $z \in \mathcal{Z}(\mathfrak{R})$ and an additive map $\Xi: \mathfrak{R} \rightarrow \mathcal{Z}(\mathfrak{R})$ such that $\varphi(x)=z x+\Xi(x)$ for all $x \in \Re$.

Remark 6. Some examples of non-prime alternative rings satisfying the assumptions " $x \mathfrak{R} \cdot e_{1}=0 \Rightarrow x=0$ and $x \mathfrak{R} \cdot e_{2}=0 \Rightarrow x=0$ " were given in [12].

\section{The proof of main result}

It is clear that " $(\boldsymbol{\beta}) \Rightarrow(\boldsymbol{\$})$ ". We will prove "(\$) $\Rightarrow(\boldsymbol{\beta})$ " by a series of Lemmas. The following Lemmas has the same hypotheses of Theorem 4 and we need these Lemmas for the proof of "( $) \Rightarrow(\mathbf{p})$ ". Thus, let us consider $e_{1}$ a nontrivial idempotent of $\Re$.

We started with the following Lemma that characterize the commutative center of an alternative ring. 


\section{Lemma 7.}

$$
\begin{aligned}
\mathcal{Z}(\mathfrak{R})= & \left\{z_{11}+z_{22}: z_{11} \in \mathfrak{R}_{11}, z_{22} \in \mathfrak{R}_{22},\right. \\
& {\left.\left[z_{11}+z_{22}, \mathfrak{R}_{12}\right]=\left[z_{11}+z_{22}, \mathfrak{R}_{21}\right]=\{0\}\right\} }
\end{aligned}
$$

Proof: On the one hand assume that $z=z_{11}+z_{12}+z_{21}+z_{22} \in \mathcal{Z}(\Re)$. Then $z e_{1}=e_{1} z$ implies $z_{12}=z_{21}=0$. Furthermore, for any $x_{12} \in \mathfrak{R}_{12}$ and $x_{21} \in \mathfrak{R}_{21}$, it follows that $z x_{12}=x_{12} z$ and $z x_{21}=x_{21} z$ that

$$
\left[z_{11}+z_{22}, \mathfrak{R}_{12}\right]=\left[z_{11}+z_{22}, \mathfrak{R}_{21}\right]=\{0\} .
$$

On the other hand, assume that $z_{11} \in \mathfrak{R}_{11}, z_{22} \in \mathfrak{R}_{22}$, and

$$
\left[z_{11}+z_{22}, \mathfrak{R}_{12}\right]=\left[z_{11}+z_{22}, \mathfrak{R}_{21}\right]=\{0\} .
$$

To prove $z_{11}+z_{22} \in \mathcal{Z}(\Re)$, one only needs to check $z_{i i} \in \mathcal{Z}\left(\Re_{i i}\right), i=1,2$. In fact, for any $r_{11} \in \mathfrak{R}_{11}$ and any $r_{12} \in \mathfrak{R}_{12}$, we have

$$
\begin{gathered}
\left(z_{11} r_{11}-r_{11} z_{11}\right) r_{12}=\left(z_{11} r_{11}\right) r_{12}-\left(r_{11} z_{11}\right) r_{12}=z_{11}\left(r_{11} r_{12}\right)-r_{11}\left(z_{11} r_{12}\right)= \\
\left(r_{11} r_{12}\right) z_{22}-r_{11}\left(r_{12} z_{22}\right)=r_{11}\left(r_{12} z_{22}\right)-r_{11}\left(r_{12} z_{22}\right)=0 .
\end{gathered}
$$

Hence $\left(z_{11} r_{11}-r_{11} z_{11}\right) \mathfrak{R} \cdot e_{2}=0$. Therefore $z_{11} \in \mathcal{Z}\left(\Re_{11}\right)$ by condition of Theorem 4. Similarly, we can check $z_{22} \in \mathcal{Z}\left(\Re_{22}\right)$.

Now we give the following lemma which plays a crucial role in this paper.

Lemma 8. For $z_{i i} \in \mathcal{Z}\left(\Re_{i i}\right), i=1,2$, there exists an element $z \in \mathcal{Z}(\Re)$ such that $z_{i i}=z e_{i}$.

Proof: Since $\mathfrak{R}$ is 3 -torsion free alternative ring we get $\mathcal{Z}\left(\mathfrak{R}_{i i}\right) \subseteq$ $\mathcal{N}\left(\Re_{i i}\right)$. Let be $z_{i i} \in \mathcal{Z}\left(R_{i i}\right)$, it is clear that $e_{i} x z_{i i}=z_{i i} x e_{i}$ holds for all $x \in \mathfrak{R}$ then, by [2, Lemma 4], there is an element $z \in \mathcal{Z}(\mathfrak{R})$ such that $z_{i i}=z e_{i}$.

The next Lemma shows where idempotents of $\mathfrak{R}$ are taken by a commuting map.

Lemma 9. $\left\{\varphi(1), \varphi\left(e_{i}\right)\right\} \subset \mathfrak{R}_{11}+\mathfrak{R}_{22}$. Moreover, there exist some $z_{i} \in$ $\mathcal{Z}(\Re)$ such that $e_{i} \varphi(1) e_{i}=z_{i} e_{i}$ with $i \in\{1,2\}$.

Proof: For any $x \in \mathfrak{R}$, we have $[\varphi(x+1), x+1]=0$. Hence

$$
0=[\varphi(x+1), x+1]=[\varphi(x+1), x]=[\varphi(1), x]
$$

holds for all $x \in \mathfrak{R}$. Particularly, $\left[\varphi(1), e_{1}\right]=0$ and $\varphi(1) \in \mathfrak{R}_{11}+\mathfrak{R}_{22}$. As $0=\left[\varphi(1), x_{i i}\right]=\left[e_{i} \varphi(1) e_{i}, x_{i i}\right]$ for all $x_{i i} \in \mathfrak{R}_{i i}$. These imply $e_{i} \varphi(1) e_{i} \in$ $\mathcal{Z}\left(\Re_{i i}\right)$, that is, there exists $z_{i} \in \mathcal{Z}(\Re)$ such that $e_{i} \varphi(1) e_{i}=z_{i} e_{i}$ with $i \in\{1,2\}$. For $e_{i}$, note that $\left[\varphi\left(e_{i}\right), e_{i}\right]=0$. It follows that $\varphi\left(e_{i}\right) \in \mathfrak{R}_{11}+\mathfrak{R}_{22}$. 
The next Lemmas 10, 11 we setting the components of Peirce's decomposition are hold by commuting map.

Lemma 10. For any $x_{i i} \in \mathfrak{R}_{i i}$, we have $\varphi\left(x_{i i}\right) \in \mathfrak{R}_{11}+\mathfrak{R}_{22}$ and there exists $z_{i} \in \mathcal{Z}(\Re)$ such that $e_{j} \varphi\left(x_{i i}\right) e_{j}=z_{i} e_{j}, 1 \leq i \neq j \leq 2$. Consequently, $e_{j} \varphi\left(e_{i}\right) e_{j}=z_{i} e_{j}$.

Proof: Taking any $x_{11} \in \mathfrak{R}_{11}$, we have

$0=\left[\varphi\left(x_{11}\right), x_{11}\right]=\left[e_{1} \varphi\left(x_{11}\right) e_{1}+e_{1} \varphi\left(x_{11}\right) e_{2}+e_{2} \varphi\left(x_{11}\right) e_{1}+e_{2} \varphi\left(x_{11}\right) e_{2}, x_{11}\right]=$ $\left[e_{1} \varphi\left(x_{11}\right) e_{1}, x_{11}\right]+\left[e_{1} \varphi\left(x_{11}\right) e_{2}, x_{11}\right]+\left[e_{2} \varphi\left(x_{11}\right) e_{1}, x_{11}\right] \in \mathfrak{R}_{11}+\mathfrak{R}_{12}+\mathfrak{R}_{21}$.

Thus,

$$
\left[e_{1} \varphi\left(x_{11}\right) e_{1}, x_{11}\right]=\left[e_{1} \varphi\left(x_{11}\right) e_{2}, x_{11}\right]=\left[e_{2} \varphi\left(x_{11}\right) e_{1}, x_{11}\right]=0
$$

holds for any $x_{11} \in \mathfrak{R}_{11}$. It follows that $\left(x_{11}+e_{1}\right) e_{1} \varphi\left(x_{11}+e_{1}\right) e_{2}=0$. Since $e_{1} \varphi\left(e_{1}\right) e_{2}=0$ we get $e_{1} \varphi\left(x_{11}\right) e_{2}=0$. As $\left[e_{2} \varphi\left(x_{11}\right) e_{1}, x_{11}\right]=0$ using a similar argument to the above we have that $e_{2} \varphi\left(x_{11}\right) e_{1}=0$. Thus, we have proved that $\varphi\left(x_{11}\right) \in \mathfrak{R}_{11}+\mathfrak{R}_{22}$ and $\left[e_{1} \varphi\left(x_{11}\right) e_{1}, x_{11}\right]=0$ holds for all $x_{11} \in \mathfrak{R}_{11}$. Analogously, we get $\varphi\left(x_{22}\right) \in \mathfrak{R}_{11}+\mathfrak{R}_{22}$ and $\left[e_{2} \varphi\left(x_{22}\right) e_{2}, x_{22}\right]=0$ holds for all $x_{22} \in \mathfrak{R}_{22}$. Now note that

$$
0=\left[\varphi\left(x_{11}+x_{22}\right), x_{11}+x_{22}\right]=\left[e_{2} \varphi\left(x_{11}\right) e_{2}, x_{22}\right]+\left[e_{1} \varphi\left(x_{22}\right) e_{1}, x_{11}\right],
$$

and so

$$
\left[e_{2} \varphi\left(x_{11}\right) e_{2}, x_{22}\right]=\left[e_{1} \varphi\left(x_{22}\right) e_{1}, x_{11}\right]=0 .
$$

It follows that $e_{j} \varphi\left(x_{i i}\right) e_{j} \in \mathcal{Z}\left(\Re_{j j}\right)$ and by Lemma 8 we obtain $e_{j} \varphi\left(x_{i i}\right) e_{j}=$ $z_{i} e_{j}$, where $z_{i} \in \mathcal{Z}(\Re), 1 \leq i \neq j \leq 2$.

Lemma 11. For any $x_{i j} \in \mathfrak{R}_{i j}$ with $1 \leq i \neq j \leq 2$ we have

(i) $e_{j} \varphi\left(x_{i j}\right) e_{i}=0$;

(ii) $e_{i} \varphi\left(x_{i j}\right) e_{j}=\left[e_{i} \varphi\left(e_{i}\right) e_{i}+e_{j} \varphi\left(e_{i}\right) e_{j}, x_{i j}\right]=-\left[e_{j} \varphi\left(e_{j}\right) e_{j}+e_{i} \varphi\left(e_{j}\right) e_{i}, x_{i j}\right]$;

(iii) $\left[e_{i} \varphi\left(x_{i j}\right) e_{i}+e_{j} \varphi\left(x_{i j}\right) e_{j}, x_{i j}\right]=0$;

(iv) $e_{i} \varphi\left(x_{i j}\right) e_{i}=z e_{i}$ and $e_{j} \varphi\left(x_{i j}\right) e_{j}=z^{\prime} e_{j}$ with $z, z^{\prime} \in \mathcal{Z}(\Re)$.

Proof: We will only prove case $i=1, j=2$ because the other the case $i=2, j=1$ has similar proof. For any $x_{12} \in \Re_{12}$ we have

$$
0=\left[\varphi\left(x_{12}\right), x_{12}\right]=e_{1} \varphi\left(x_{12}\right) e_{1} x_{12}+e_{1} \varphi\left(x_{12}\right) e_{2} x_{12}+e_{2} \varphi\left(x_{12}\right) e_{1} x_{12}
$$




$$
-x_{12} e_{1} \varphi\left(x_{12}\right) e_{2}-x_{12} e_{2} \varphi\left(x_{12}\right) e_{1}-x_{12} e_{2} \varphi\left(x_{12}\right) e_{2} .
$$

Hence we get $\left[e_{1} \varphi\left(x_{12}\right) e_{1}+e_{2} \varphi\left(x_{12}\right) e_{2}, x_{12}\right]=0$ this shows $(i i i)$ and

$$
x_{12} e_{2} \varphi\left(x_{12}\right) e_{1}=0, e_{1} \varphi\left(x_{12}\right) e_{2} x_{12}=0, e_{2} \varphi\left(x_{12}\right) e_{1} x_{12}=0 .
$$

Now we have too

$$
\begin{gathered}
0=\left[\varphi\left(x_{12}+e_{1}\right), x_{12}+e_{1}\right]= \\
e_{2} \varphi\left(x_{12}\right) e_{1}-e_{1} \varphi\left(x_{12}\right) e_{2}+e_{1} \varphi\left(e_{1}\right) e_{1} x_{12}-x_{12} e_{2} \varphi\left(e_{1}\right) e_{2} .
\end{gathered}
$$

Thus, $e_{2} \varphi\left(x_{12}\right) e_{1}=0$ that is $(i)$ and

$$
e_{1} \varphi\left(x_{12}\right) e_{2}=\left[e_{1} \varphi\left(e_{1}\right) e_{1}+e_{2} \varphi\left(e_{1}\right) e_{2}, x_{12}\right] .
$$

As $\left[\varphi\left(x_{12}+e_{2}\right), x_{12}+e_{2}\right]=0$ by a straightforward calculation follows that

$$
e_{1} \varphi\left(x_{12}\right) e_{2}=-\left[e_{2} \varphi\left(e_{2}\right) e_{2}+e_{1} \varphi\left(e_{2}\right) e_{1}, x_{12}\right]
$$

Of (11) and (2) we obtain (ii). We still need to proof $(i v)$. For this to observe that $\left[e_{1} \varphi\left(x_{11}\right) e_{1}, x_{11}\right]=0$ by the Lemma 10. Since

$$
\left[\varphi\left(x_{11}+x_{12}\right), x_{11}+x_{12}\right]=0 \text { and }\left[\varphi\left(x_{12}\right), x_{11}\right] \in \mathfrak{R}_{11}+\mathfrak{R}_{12}
$$

and using the identities $(i)$ and $($ iii) we conclude

$$
\left[\varphi\left(x_{12}\right), x_{11}\right]=0 \text { and }\left[e_{1} \varphi\left(x_{12}\right) e_{1}, x_{11}\right]=0 .
$$

Therefore, by Lemma 8 there exists $z \in \mathcal{Z}(\mathfrak{R})$ such that $e_{1} \varphi\left(x_{12}\right) e_{1}=z e_{1}$ that is $(i v)$. The proof is complete.

In addition to Lemma 9 the next Lemma we showed specifically where commuting map takes the unity of $\mathfrak{R}$.

Lemma 12. $\varphi(1) \in \mathcal{Z}(\Re)$, and, there exists $z_{i} \in \mathcal{Z}(\mathfrak{R})$ such that $e_{i} \varphi\left(e_{i}\right) e_{i}=$ $z_{i} e_{i} \in \mathcal{Z}\left(\Re_{i i}\right), i=1,2$.

Proof: By Lemmas 9 and 10, we have

$$
e_{1} \varphi\left(e_{1}\right) e_{1}=e_{1} \varphi(1) e_{1}-e_{1} \varphi\left(e_{2}\right) e_{1}=(\alpha-\beta) e_{1}=z_{1} e_{1} \in \mathcal{Z}\left(\Re_{11}\right)
$$

and

$$
e_{2} \varphi\left(e_{2}\right) e_{2}=e_{2} \varphi(1) e_{2}-e_{2} \varphi\left(e_{1}\right) e_{2}=(\lambda-\mu) e_{1}=z_{2} e_{1} \in \mathcal{Z}\left(\Re_{22}\right),
$$

where $\alpha, \beta, \lambda, \mu \in \mathcal{Z}(\Re)$ and $(\alpha-\beta)=z_{1},(\lambda-\mu)=z_{2}$.

By Lemma 11 items $(i i)$ and $($ iii) we get

$$
\left[e_{1} \varphi\left(e_{1}\right) e_{1}+e_{2} \varphi\left(e_{1}\right) e_{2}, x_{12}\right]=-\left[e_{2} \varphi\left(e_{2}\right) e_{2}+e_{1} \varphi\left(e_{2}\right) e_{1}, x_{12}\right],
$$

hence $\left[\varphi(1), x_{12}\right]=\left[\varphi\left(e_{1}\right)+\varphi\left(e_{2}\right), x_{12}\right]=0$. Similarly, $\left[\varphi(1), x_{21}\right]=0$. It follows that $\varphi(1) \in \mathcal{Z}(\Re)$ by Lemma 7 . 
And finally, the next Lemmas 13, 16 tells us the behavior to the image of Peirce's components by commuting map.

Lemma 13. There exists $z, z^{\prime} \in \mathcal{Z}(\mathfrak{R})$ such that $e_{1} \varphi\left(e_{1}\right) e_{1}+e_{2} \varphi\left(e_{2}\right) e_{2}-$ $\left(z e_{1}+z^{\prime} e_{2}\right) \in \mathcal{Z}(\Re)$.

Proof: By Lemma 12 we have

$$
e_{1} \varphi\left(e_{1}\right) e_{1}-z e_{1} \in \mathcal{Z}\left(\Re_{11}\right) \text { and } e_{2} \varphi\left(e_{2}\right) e_{2}-z^{\prime} e_{2} \in \mathcal{Z}\left(\Re_{22}\right) .
$$

Moreover, since $\varphi(1) \in \mathcal{Z}(\mathfrak{R})$ and Lemma 10 we get

$$
\begin{gathered}
e_{1} \varphi\left(e_{1}\right) e_{1} x_{12}+x_{12}\left(z^{\prime} e_{2}\right)=\left(e_{1} \varphi\left(e_{1}\right) e_{1}+z^{\prime} e_{2}\right) x_{12}= \\
\left(e_{1} \varphi\left(e_{1}\right) e_{1}+e_{1} \varphi\left(e_{2}\right) e_{1}\right) x_{12}=x_{12}\left(e_{2} \varphi\left(e_{1}\right) e_{2}+e_{2} \varphi\left(e_{2}\right) e_{2}\right)= \\
x_{12}\left(z e_{2}+e_{2} \varphi\left(e_{2}\right) e_{2}\right)=z e_{1} x_{12}+x_{12} e_{2} \varphi\left(e_{2}\right) e_{2},
\end{gathered}
$$

that is, $\left[\left(e_{1} \varphi\left(e_{1}\right) e_{1}-z e_{1}\right)+\left(e_{2} \varphi\left(e_{2}\right) e_{2}-z^{\prime} e_{2}\right), x_{12}\right]=0$ for all $x_{12} \in \mathfrak{R}_{12}$. Similarly, $\left[\left(e_{1} \varphi\left(e_{1}\right) e_{1}-z e_{1}\right)+\left(e_{2} \varphi\left(e_{2}\right) e_{2}-z^{\prime} e_{2}\right), x_{21}\right]=0$ for all $x_{21} \in \mathfrak{R}_{21}$. By Lemma 7 we conclude $\left(e_{1} \varphi\left(e_{1}\right) e_{1}-z e_{1}\right)+\left(e_{2} \varphi\left(e_{2}\right) e_{2}-z^{\prime} e_{2}\right) \in \mathcal{Z}(\Re)$.

Lemma 14. We have $\left[e_{1} \varphi\left(x_{i j}\right) e_{1}+e_{2} \varphi\left(x_{i j}\right) e_{2}, x_{j i}\right]=0$ for all $x_{i j} \in \mathfrak{R}_{i j}$ and $x_{j i} \in \mathfrak{R}_{j i}$ with $i \neq j$.

Proof: By definition of $\varphi$ we have $\left[\varphi\left(1+x_{12}+x_{21}\right), 1+x_{12}+x_{21}\right]=0$. Using Lemmas 11 and 12 we get

$$
\left[\varphi\left(1+x_{12}+x_{21}\right), 1+x_{12}+x_{21}\right]=\left[\varphi\left(x_{12}\right), x_{21}\right]+\left[\varphi\left(x_{21}\right), x_{12}\right],
$$

which implies that

$$
\left[e_{1} \varphi\left(x_{12}\right) e_{1}+e_{2} \varphi\left(x_{12}\right) e_{2}, x_{21}\right]=\left[e_{1} \varphi\left(x_{21}\right) e_{1}+e_{2} \varphi\left(x_{21}\right) e_{2}, x_{12}\right]=0
$$

for all $x_{12} \in \mathfrak{R}_{12}$ and $x_{21} \in \mathfrak{R}_{21}$.

Lemma 15. We have $\left[e_{i} \varphi\left(x_{i j}\right) e_{i}+e_{j} \varphi\left(x_{i j}\right) e_{j}, r\right]=0$ for all $r \in \mathfrak{R}$ and $x_{i j} \in \mathfrak{R}_{i j}$ with $i \neq j$.

Proof: By Lemma 11 item (iv) we have $e_{i} \varphi\left(x_{i j}\right) e_{i}=z e_{i}$ and $e_{j} \varphi\left(x_{i j}\right) e_{j}=z^{\prime} e_{j}$, where $z, z^{\prime} \in \mathcal{Z}(\Re)$. and using Lemma 14 we get $\left[z e_{i}+z^{\prime} e_{j}, x_{j i}\right]=0$, that implies

$$
\left(z^{\prime} e_{j}-z e_{j}\right) x \cdot e_{i}=0,
$$

for all $x \in \mathfrak{R}$. Thus by assumption of Theorem 4 we have $z e_{j}=z^{\prime} e_{j}$. Therefore

$$
\left[e_{i} \varphi\left(x_{i j}\right) e_{i}+e_{j} \varphi\left(x_{i j}\right) e_{j}, r\right]=\left[z e_{i}+z^{\prime} e_{j}, r\right]=\left[z e_{i}+z e_{j}, r\right]=[z, r]=0
$$

for all $r \in \Re$. 
Lemma 16. We have $e_{i} \varphi\left(x_{i i}\right) e_{i}=z_{i} e_{i}+\left(e_{i} \varphi\left(e_{i}\right) e_{i}-z_{i}^{\prime} e_{i}\right) x_{i i}$ for all $x_{i i} \in \Re_{i i}$ with $z_{i}, z_{i}^{\prime} \in \mathcal{Z}(\Re)$ and $i \in\{1,2\}$.

Proof: Consider $i \neq j$ with $i, j \in\{1,2\}$. Let be $x_{i i} \in \mathfrak{R}_{i i}$ and $x_{i j} \in \mathfrak{R}_{i j}$, by Lemma 12 and Lemma 11 item (iii), we get

$$
0=\left[\varphi\left(x_{i i}+x_{i j}\right), x_{i i}+x_{i j}\right]=\left[\varphi\left(x_{i i}\right), x_{i j}\right]+\left[\varphi\left(x_{i j}\right), x_{i i}\right] .
$$

It follow that

$$
e_{i} \varphi\left(x_{i i}\right) e_{i} x_{i j}-x_{i j} e_{j} \varphi\left(x_{i i}\right) e_{j}-x_{i i} e_{i} \varphi\left(x_{i j}\right) e_{j}=0,
$$

where have used Lemma 15 and Lemma 11 item $(i)$. By Lemma 10 to observe that $e_{j} \varphi\left(x_{i i}\right) e_{j}=z_{i} e_{j}$ with $z_{i}=z_{i 11}+z_{i 22} \in \mathcal{Z}(\mathfrak{R})$ since

$$
x_{i j} \cdot\left(z_{i 11}+z_{i 22}\right) e_{j}=\left(z_{i 11}+z_{i 22}\right) e_{i} \cdot x_{i j}
$$

and by Lemma 11 item (ii) we have

$x_{i i} e_{i} \varphi\left(x_{i j}\right) e_{j}=x_{i i} \cdot e_{i} \varphi\left(e_{i}\right) e_{i} x_{i j}-x_{i i} \cdot z_{i}^{\prime} e_{i} x_{i j}=x_{i i} e_{i} \varphi\left(e_{i}\right) e_{i} \cdot x_{i j}-x_{i i} z_{i}^{\prime} e_{i} \cdot x_{i j}$,

with $z_{i}^{\prime} \in \mathcal{Z}(\mathfrak{R})$. Hence we can write (3) as

$$
e_{i} \varphi\left(x_{i i}\right) e_{i} x_{i j}-z_{i} e_{i} \cdot x_{i j}-x_{i i} e_{i} \varphi\left(e_{i}\right) e_{i} \cdot x_{i j}+x_{i i} z_{i}^{\prime} e_{i} \cdot x_{i j}=0,
$$

that is,

$$
\left(e_{i} \varphi\left(x_{i i}\right) e_{i}-z_{i} e_{i}-x_{i i} e_{i} \varphi\left(e_{i}\right) e_{i}+x_{i i} z_{i}^{\prime} e_{i}\right) e_{i} x e_{j}=0 \text { for all } x \in \mathfrak{R} .
$$

By the assumption of Theorem 4 we get

$$
e_{i} \varphi\left(x_{i i}\right) e_{i}=z_{i} e_{i}+\left(e_{i} \varphi\left(e_{i}\right) e_{i}-z_{i}^{\prime} e_{i}\right) x_{i i}
$$

Now we are ready to prove "(\$) $\Rightarrow(\boldsymbol{\$})$ " of the Theorem 4 ,

Prove of the Theorem $4((\boldsymbol{\$}) \Rightarrow(\boldsymbol{\beta}))$ : Let us define $\Xi(x)=\varphi(x)-z x$ with $z=e_{1} \varphi\left(e_{1}\right) e_{1}+e_{2} \varphi\left(e_{2}\right) e_{2}-\left(z_{1} e_{1}+z_{2} e_{2}\right) \in \mathcal{Z}(\Re)$ likewise Lemma 13 . Note that $e_{j} \varphi\left(e_{i}\right) e_{j}=z_{i} e_{j}$, with $i \neq j$. It is clear that $\Xi$ is additive on $\mathfrak{R}$. Now we just need to prove that $\Xi(x) \in \mathcal{Z}(\Re)$. Let be $x=x_{11}+x_{12}+x_{21}+$ $x_{22} \in \mathfrak{R}$. By Lemmas 10 and 11 we have 


$$
\begin{aligned}
\Xi(x) & =\underbrace{e_{1} \varphi\left(x_{11}\right) e_{1}+e_{2} \varphi\left(x_{11}\right) e_{2}-e_{1} \varphi\left(e_{1}\right) e_{1}+z_{1} e_{1} x_{11}}_{\in \mathcal{Z}(\mathfrak{R}) \text { by Lemma 16 }} \\
& +\underbrace{e_{1} \varphi\left(x_{22}\right) e_{1}+e_{2} \varphi\left(x_{22}\right) e_{2}-e_{2} \varphi\left(e_{2}\right) e_{2} x_{22}+z_{2} e_{2} x_{22}}_{\in \mathcal{Z}(\Re) \text { by Lemma 16 }} \\
& +\underbrace{e_{1} \varphi\left(x_{12}\right) e_{1}+e_{2} \varphi\left(x_{12}\right) e_{2}+e_{1} \varphi\left(x_{21}\right) e_{1}+e_{2} \varphi\left(x_{21}\right) e_{2}}_{\in \mathcal{Z}(\Re) \text { by Lemma 15 } 15} \\
& +\underbrace{z_{1} e_{1} \cdot x_{12}-x_{12} e_{2} \varphi\left(e_{1}\right) e_{2}+z_{2} e_{2} \cdot x_{21}-x_{21} e_{1} \varphi\left(e_{2}\right) e_{1}}_{\in \mathcal{Z}(\Re) \text { by Lemma } 7} .
\end{aligned}
$$

Therefore the proof $(\mathbf{M}) \Rightarrow(\boldsymbol{\$})$ of the Theorem 4 is complete.

Definition 17. A nonlinear map $\varphi: \mathfrak{R} \rightarrow \mathfrak{R}$ is called anti-commuting if $[\varphi(a), b]=-[a, \varphi(b)]$ holds for all $a, b \in \mathfrak{R}$.

Corollary 18. Let $\mathfrak{R}$ be a unital 2,3-torsion free alternative ring. Assume that $\mathfrak{R}$ has a nontrivial idempotent $e_{1}$ with associated Peirce decomposition $\mathfrak{R}=\mathfrak{R}_{11} \oplus \mathfrak{R}_{12} \oplus \mathfrak{R}_{21} \oplus \mathfrak{R}_{22}$, such that $x \mathfrak{R} \cdot e_{i}=0 \Rightarrow x=0(i=1,2)$. Let $\varphi: \mathfrak{R} \rightarrow \mathfrak{R}$ be an additive map. Then the following statements are equivalent:

(\$) $\varphi$ is anti-commuting;

(\$) There exist $z \in \mathcal{Z}(\mathfrak{R})$ and an additive map $\Xi: \mathfrak{R} \rightarrow \mathcal{Z}(\mathfrak{R})$ such that $\varphi(x)=z x+\Xi(x)$ for all $x \in \mathfrak{R}$.

\section{Application on alternative $C^{*}$-algebras}

Recall that an alternative $C^{*}$-algebra $\mathcal{U}$ is a complete normed alternative complex algebra endowed with a conjugate-linear algebra involution * satisfying $\left\|a^{*} a\right\|=\|a\|^{2}$ for every $a \in \mathcal{U}$. It is well known that alternative $C^{*}$-algebra admits representation as a von Neumann algebra. Let be $\mathcal{M}_{\mathcal{U}}$ the von Neumann algebra relative alternative $C^{*}$-algebra $\mathcal{U}$. For those readers who are not familiar with this language of alternative $C^{*}$-algebra we recommend [15, 16]. It is shown [2] that, if a von Neumann algebra $\mathcal{M}$ has no central summands of type $I_{1}$ (= central abelian projection), then $\mathcal{M}$ satifies the follow assumptions

- $X \mathcal{M} \cdot e_{1}=\{0\} \Rightarrow X=0$, 
- $X \mathcal{M} \cdot e_{2}=\{0\} \Rightarrow X=0$.

Therefore, we have the follow result

Corollary 19. Let $\mathcal{M}_{\mathcal{U}}$ be a von Neumann algebra relative alternative $C^{*}$ algebra $\mathcal{U}$ and $\varphi: \mathcal{M}_{\mathcal{U}} \rightarrow \mathcal{M}_{\mathcal{U}}$ be an additive map. Then the following statements are equivalent:

(\$) $\varphi$ is commuting;

(3) There exist $Z \in \mathcal{Z}\left(\mathcal{M}_{\mathcal{U}}\right)$ and an additive map $\Xi: \mathcal{M}_{\mathcal{U}} \rightarrow \mathcal{Z}\left(\mathcal{M}_{\mathcal{U}}\right)$ such that $\varphi(X)=Z X+\Xi(X)$ for all $X \in \mathcal{M}_{\mathcal{U}}$.

Proof: Since $\mathcal{M}_{\mathcal{U}}$ has no central summands of type $I_{1}$, then there exists idempotent $e_{i} \in \mathcal{M}_{\mathcal{U}}$ satisfying $X \mathcal{M}_{\mathcal{U}} \cdot e_{i}=\{0\} \Rightarrow X=0$. Now we just need to prove $e_{i} X e_{i} \in \mathcal{Z}\left(\mathcal{M}_{\mathcal{U}_{i i}}\right)$, there exist $e_{j} X e_{j} \in \mathcal{M}_{\mathcal{U}_{j j}}$ such that $e_{i} X e_{i}+e_{j} X e_{j} \in \mathcal{Z}\left(\mathcal{M}_{\mathcal{U}}\right)$ with $1 \leq i \neq j \leq 2$. Let be any $e_{i} X e_{i} \in \mathcal{Z}\left(\mathcal{M}_{\mathcal{U}_{i i}}\right)$ so by Lemma 4 in [2] there is an element $Z \in \mathcal{Z}\left(\mathcal{M}_{\mathcal{U}}\right)$ such that $e_{i} X e_{i}=$ $Z e_{i}$. Hence there exist $e_{j} X e_{j}=e_{j} Z e_{j} \in \mathcal{M}_{\mathcal{U} j j}$ such that $e_{i} X e_{i}+e_{j} X e_{j} \in$ $\mathcal{Z}\left(\mathcal{M}_{\mathcal{U}}\right)$. Now, by Theorem 4 , the corollary is true.

\section{References}

[1] Albert A., Quadratic forms permitting composition, Annals of Mathematics, 43 (1942), 161-177.

[2] Bai Z., Du S., Strong commutativity preserving maps on rings, Rocky Mountain Journal of Mathematics, 44 (2014), 733-742.

[3] Bresar M., Centralizing mappings on von Neumann algebras, Proceedings of the American Mathematical Society, 111 (1991), 501-510.

[4] Bresar M., Commuting traces of biadditive mappings, commutativitypreserving mappings and Lie mappings, Transactions of the American Mathematical Society, 335 (1993), 525-546.

[5] Bresar M., Centralizing mappings and derivations in prime rings, Journal of Algebra, 156 (1993), 385-394.

[6] Bresar M., Commuting maps: a survey, Taiwanese Journal of Mathematics, 8 (2004), 361-397.

[7] Cheung W.-S., Commuting maps of triangular algebras, Journal of the London Mathematical Society, 63 (2001), 117-127. 
[8] Divinsky N., On commuting automorphisms of rings, Transactions of the Royal Society of Canada III, 49 (1955), 19-22.

[9] Ferreira J.C.M., Ferreira B.L.M., Additivity of $n$-multiplicative maps on alternative rings, Communications in Algebra, 44 (2016), 1557-1568.

[10] Ferreira R. N., Ferreira B.L.M., Jordan triple derivation on alternative rings, Proyecciones Journal of Mathematics, 37 (2018), 171-180.

[11] Ferreira B.L.M., Guzzo H., Characterizaiton of Lie multiplicative derivation of alternative Rings, Rocky Mountain Journal of Mathematics, 49 (2019), 761-772.

[12] Ferreira B.L.M., Guzzo H., Lie Maps on alternative rings, Bollettino dell'Unione Matematica Italiana, 13 (2020), 2, 181-192.

[13] Ferreira B.L.M., Guzzo H., Kaygorodov I., Lie maps on alternative rings preserving idempotents, arXiv:2003.03371

[14] Ferreira B.L.M., Guzzo H., Wei F., Multiplicative Lie-type derivations on alternative rings, arXiv:2002.00304.

[15] García M. C., Palacios Á. R., Non-associative normed algebras. Vol. 1. The Vidav-Palmer and Gelfand-Naimark theorems. Encyclopedia of Mathematics and its Applications, 154. Cambridge University Press, Cambridge, 2014. xxii+712 pp.

[16] García M. C., Palacios Á. R., Non-associative normed algebras. Vol. 2. Representation theory and the Zel'manov approach. Encyclopedia of Mathematics and its Applications, 167. Cambridge University Press, Cambridge, 2018. xxvii+729 pp.

[17] Hentzel I., Kleinfeld E., Smith H., Alternative rings with idempotent, Journal of Algebra, 64 (1980), 325-335.

[18] Kaygorodov I., Popov Yu., Alternative algebras that admit derivations with invertible values and invertible derivations, Izvestiya: Mathematics, 78 (2014), 5, 922-936.

[19] Lee P.-H., Wang Y., Supercentralizing maps on prime superalgebras, Communications in Algebra, 37 (2009), 840-854.

[20] Li C.-J., Fang X.-C., Lu F.-Y., Wang T., Lie triple derivable mappings on rings, Communications in Algebra, 42 (2014), 2510-2527. 